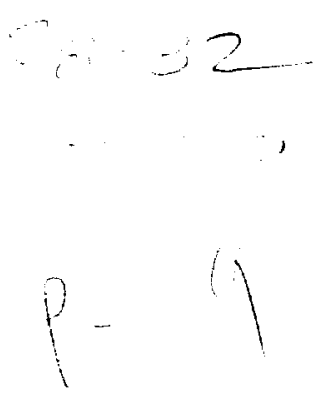

\title{
Maximum and Minimum Return Losses From a Passive Two-Port Network Terminated With a Mismatched Load
}

\author{
T. Y. Otoshi \\ Ground Antennas and Facilities Engineering Section
}

\begin{abstract}
This article presents an analytical method for determining the exact distance a load is required to be offset from a passive two-port network to obtain maximum or minimum return losses from the terminated two-port network. Equations are derived in terms of two-port network $S$-parameters and load reflection coefficient. The equations are useful for predicting worst-case performances of some types of networks that are terminated with offset short-circuit loads.
\end{abstract}

\section{Introduction}

With the advent of automatic network analyzers, it has become common practice to characterize microwave twoport networks in terms of measured S-parameters [1]. As a result, many measurement techniques and network configurations are now being analyzed in terms of S-parameters rather than in terms of the impedance and admittance characterization parameters that have been used in the past. Microwave measurement techniques often employ the use of a sliding load, sliding short, or mismatched loads of known reflection-coefficient values. A frequently encountered problem of interest is determining the distance a particular load has to be offset from the two-port network output port to obtain a maximum or minimum return loss from the terminated two-port network. When the load is fixed (rather than sliding), line spacers can be inserted between the output of the two-port network and the load for producing the desired return losses. It is desirable to know what spacer-line length is required without having to resort to a trial-and-error process. A literature search indicates that analytical solutions, in terms of Sparameters, have not been published for these purposes.
This article will present these analytical expressions of interest.

Section II of this article sets forth the theory and analytical derivations of the conditions for maximum and minimum return losses. Also presented are design equations for dielectric spacers that can be used to obtain desired return losses. Section III presents examples showing how these equations can be used for predicting and avoiding the occurrence of a type of resonance phenomenon (leading to large dissipative losses) for some types of reflector antennas.

\section{Theory}

\section{A. Derivations}

The circuit under consideration is shown in Fig. 1 and the following conditions exist. It is assumed that the Sparameters of the two-port network and load reflectioncoefficient values are known. In practice, if the load is fixed, the phase of its reflection coefficient as defined at the network output port can be varied through the use of 
dielectric spacers. The following presents the derivation of equations required to calculate the specific conditions under which maximum or minimum return losses will be obtained from the terminated network.

Given the two-port network terminated in $\Gamma_{L}$ as shown in Fig. 1, the input reflection coefficient as seen looking into port 1 is

$$
\Gamma_{1}=S_{11}+\frac{S_{12} S_{21} \Gamma_{L}}{1-S_{22} \Gamma_{L}}
$$

where $S_{11}, S_{12}, S_{21}$, and $S_{22}$ are scattering parameters [1]. From Eq. (1),

$$
\left|\Gamma_{1}\right|=\sqrt{\frac{\left|S_{11}\right|^{2}+2\left|S_{11} U \Gamma_{L}\right| \cos \theta_{1}+\left|U \Gamma_{L}\right|^{2}}{1-2\left|S_{22} \Gamma_{L}\right| \cos \theta_{2}+\left|S_{22} \Gamma_{L}\right|^{2}}}
$$

where

$$
\begin{gathered}
U=S_{12} S_{21}-S_{11} S_{22} \\
\theta_{1}=\psi_{L}+\psi_{U}-\psi_{11} \\
\theta_{2}=\psi_{L}+\psi_{22}
\end{gathered}
$$

and $\psi_{L}, \psi_{U}, \psi_{11}$, and $\psi_{22}$ are the phase angles of $\Gamma_{L}, U$, $S_{11}$, and $S_{22}$, respectively.

-In the following analysis, $\left|\Gamma_{L}\right|$ is assumed to be a constant and only $\psi_{L}$ is allowed to vary. To find the maximum or minimum values of $\left|\Gamma_{1}\right|$ under these conditions, set

$$
\frac{\partial\left|\Gamma_{1}\right|^{2}}{\partial \psi_{L}}=0
$$

This leads to an equation of the form

$$
A \sin \theta_{1}+B \sin \theta_{2}+C=0
$$

where

$$
\begin{gathered}
A=\left|S_{11} U\right|\left(1+\left|S_{22} \Gamma_{L}\right|^{2}\right) \\
B=\left|S_{22}\right|\left(\left|S_{11}\right|^{2}+\left|U \Gamma_{L}\right|^{2}\right)
\end{gathered}
$$

$$
C=2\left|S_{11} S_{22} U \Gamma_{L}\right| \sin \left(\psi_{22}+\psi_{11}-\psi_{U}\right)
$$

Substituting Eqs. (4) and (5) into Eq. (7), using trigonometric sum angle identities, and collecting terms leads to the expression

$$
D \cos \psi_{L}+E \sin \psi_{L}+C=0
$$

where

$$
\begin{aligned}
& D=A \sin \left(\psi_{U}-\psi_{11}\right)+B \sin \psi_{22} \\
& E=A \cos \left(\psi_{U}-\psi_{11}\right)+B \cos \psi_{22}
\end{aligned}
$$

Upon expressing Eq. (11) in the equivalent form

$$
\sqrt{D^{2}+E^{2}} \cos \left(\psi_{L}-\varphi\right)=-C
$$

where

$$
\varphi=\tan ^{-1}\left(\frac{E}{D}\right)
$$

then, from Eq. (14), the values of $\psi_{L}$ that correspond to maximum or minimum values of $\left|\Gamma_{1}\right|$ are

$$
\psi_{L m}=\varphi \pm \cos ^{-1}\left(\frac{-C}{\sqrt{D^{2}+E^{2}}}\right)
$$

Substitution of $\psi_{L}=\psi_{L m}$ into Eqs. (4), (5), and (2) gives the desired values of $\left|\Gamma_{1}\right|_{\max }$ and $\left|\Gamma_{1}\right|_{\min }$. Return losses are computed from

$$
R L_{d B}=-20 \log _{10}\left|\Gamma_{1}\right|
$$

\section{B. Design of Spacers}

As shown in Fig. 1, if the actual load reflection coefficient is $\Gamma_{T}$, but a particular $\Gamma_{L}$ load reflection coefficient is desired to obtain a maximum or minimum return loss, a dielectric spacer can be utilized. The relationship for the dielectric spacer length of a nonferrous material is given as follows:

$$
\ell=\frac{1}{2 \beta} \arg R
$$

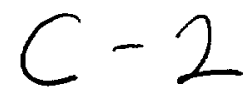


where $\beta$ is the phase constant whose expression will follow and arg $R$ is the phase angle of a complex value $R$

$$
R=\left(\frac{\Gamma_{D}+\Gamma_{T}}{1+\Gamma_{D} \Gamma_{T}}\right)\left(\frac{1+\Gamma_{D} \Gamma_{L}}{\Gamma_{D}+\Gamma_{L}}\right)
$$

Eq. (19) was derived from input impedance and reflection coefficient relationships, starting at termination and transforming through the dielectric to the $\mathrm{Z}_{01}$ input interface.

Defining $\epsilon^{\prime}$ to be the relative permittivity, $\tan \delta$ the loss tangent, $\lambda_{0}$ the free-space wavelength, and $\theta_{i}$ the incident angle, then the dielectric-air interface reflection coefficient for perpendicular polarization is

$$
\Gamma_{D}=\left(\Gamma_{D}\right)_{\perp}=\frac{\sqrt{\left(\epsilon^{\prime}-\sin ^{2} \theta_{i}\right)-j \epsilon^{\prime \prime}}-\cos \theta_{i}}{\sqrt{\left(\epsilon^{\prime}-\sin ^{2} \theta_{i}\right)-j \epsilon^{\prime \prime}}+\cos \theta_{i}}
$$

and for parallel polarization,

$$
\begin{aligned}
\Gamma_{D} & =\left(\Gamma_{D}\right)_{\|} \\
& =\frac{\left(\epsilon^{\prime}-j \epsilon^{\prime \prime}\right) \cos \theta_{i}-\sqrt{\left(\epsilon^{\prime}-\sin ^{2} \theta_{i}\right)-j \epsilon^{\prime \prime}}}{\left(\epsilon^{\prime}-j \epsilon^{\prime \prime}\right) \cos \theta_{i}+\sqrt{\left(\epsilon^{\prime}-\sin ^{2} \theta_{i}\right)-j \epsilon^{\prime \prime}}}
\end{aligned}
$$

For either polarization, the following relationships hold:

$$
\begin{gathered}
\epsilon^{\prime \prime}=\epsilon^{\prime} \tan \delta \\
\gamma=\alpha+j \beta \\
\alpha=\frac{2 \pi}{\lambda_{0}} \sqrt{\epsilon^{\prime}-\sin ^{2} \theta_{i}} \sqrt{\frac{\sqrt{1+\tan ^{2} \delta^{\prime}}-1}{2}} \\
\beta=\frac{2 \pi}{\lambda_{0}} \sqrt{\epsilon^{\prime}-\sin ^{2} \theta_{i}} \sqrt{\frac{\sqrt{1+\tan ^{2} \delta^{\prime}}+1}{2}} \\
\tan \delta^{\prime}=\frac{\epsilon^{\prime \prime}}{\epsilon^{\prime}-\sin ^{2} \theta_{i}}
\end{gathered}
$$

When measurements are made in a $T E_{10}$ mode waveguide, in the above equations for perpendicular polarization, one may substitute

$$
\begin{gathered}
\sin \theta_{i}=\frac{\lambda_{0}}{\lambda_{c}} \\
\cos \theta_{i}=\sqrt{1-\left(\frac{\lambda_{0}}{\lambda_{c}}\right)^{2}}
\end{gathered}
$$

where $\lambda_{c}$ is the cutoff wavelength for the $T E_{10}$ mode. For a dielectric sheet of thickness $\ell$, the general-case S-parameter relationships that apply to perpendicular or parallel polarization in free space or to the $T E_{10}$ mode waveguide cases are

$$
\begin{gathered}
\left(S_{11}\right)_{D}=\left(S_{22}\right)_{D}=\frac{-\Gamma_{D}\left(1-e^{-2 \gamma \ell}\right)}{1-\Gamma_{D}^{2} e^{-2 \gamma \ell}} \\
\left(S_{12}\right)_{D}=\left(S_{21}\right)_{D}=\frac{\left(1-\Gamma_{D}^{2}\right) e^{-\gamma \ell}}{1-\Gamma_{D}^{2} e^{-2 \gamma \ell}}
\end{gathered}
$$

The above formulas were derived by considering the airto-dielectric interfaces as separate individual two-port networks, and the dielectric media of length $\ell$ as the third twoport network. Then, the overall S-parameters for three cascaded networks were derived using cascading formulas given in [1].

\section{Applications}

\section{A. Salisbury Screen}

An application of the previously derived equations is the determination of maximum return loss that will occur when a perforated plate or mesh is terminated with an offset short circuit. For this type of configuration, sometimes referred to as a Salisbury Screen [2], it is of interest to know the exact offset distance, between the short and mesh, that will lead to maximum return loss. Maximum return loss for this type of configuration will occur when there is maximum dissipation due to the resistive losses of the perforated screen or mesh.

To demonstrate the application of the derived equations, Table 1 presents measured S-parameters of a perforated plate that has been used for the antenna reflector surface material on some DSN antennas. These measured S-parameters also agree well with those calculated from theory [3]. Maximum and minimum return losses for an offset short-circuit load, as calculated through Eqs. (16) and (17), are shown in Table 2. 
It is of interest to study the characteristics of this perforated plate material when terminated in an offset shortcircuit load and the offset distance is allowed to vary. Return losses calculated from Eq. (1) are plotted in Fig. 2. A sharp resonance is caused by the resistive losses of the perforated plate, and the maximum return loss is $33.2 \mathrm{~dB}$. If the perforated plate were lossless, then the return loss would have been $0 \mathrm{~dB}$. The exact short-circuit load position corresponding to the $33.2-\mathrm{dB}$ maximum return loss was calculated from equations in this article and is shown in Table 2. The resonance phenomenon shown in Fig. 2 was observed experimentally when a sample of the perforated plate test sample was inserted in the waveguide and terminated with a sliding short-circuit load. Knowledge of maximum return loss can be used to obtain information on the resistive losses of the perforated plate. Similar techniques can be applied to the determination of resistive losses of other types of meshes such as a tricot-mesh material used for spacecraft unfurlable antennas $[4,5]$.

The equations presented in this article are not limited to short-circuit loads, but are applicable for any load whose reflection-coefficient magnitude is greater than zero. For interest, Table 2 tabulates the conditions under which maximum and minimum return losses occur when the described perforated plate is terminated with loads having various load reflection-coefficient magnitudes.

\section{B. Water Film on the Reflector Surface}

The manufacturing process of some commercial Kuband reflectors involves pouring a fiberglass epoxy mix onto a thin metallic screen laid on a parabolic-shaped mold. After drying, the reflector consists of a fiberglass layer bonded to the metal screen. It will be shown that a fiberglass layer thickness of $1.59 \mathrm{~mm}$ (0.0625 in.), which sometimes results in the manufacturing process, leads to poor reflector performance at $12 \mathrm{GHz}$ when the surface becomes wet due to condensation or rain.

For theoretical calculation purposes, it will be assumed that the fiberglass dielectric material has a relative permittivity of 4.5 and loss tangent of 0.002 at $12 \mathrm{GHz}$. First, the S-parameters of the fiberglass sheet of $1.59-\mathrm{mm}$ thickness are calculated from Eqs. (29) and (30) for a normally incident, linearly polarized wave at $12 \mathrm{GHz}$. Then, the input reflection coefficient is calculated from Eq. (1) for the fiberglass sheet terminated in a short-circuit load. The result is an input reflection coefficient of 0.9994 with a phase angle of $123.9 \mathrm{deg}$ when this fiberglass sheet is terminated with a short-circuit load. The corresponding return loss value of $0.005 \mathrm{~dB}$ calculated from Eq. (17) shows that this reflector with the described fiberglass layer is an excellent reflector under dry conditions.
For purposes of analyzing the wet surface condition, a thin water film will be treated as a two-port network whose S-parameters are calculated from Eqs. (29) and (30) for water-film thicknesses varying between $0.05 \mathrm{~mm}(0.002 \mathrm{in}$.) and $0.10 \mathrm{~mm}(0.004 \mathrm{in}$.). These thicknesses are typical for a water film lying on a flat, smooth surface. A relative permittivity of 55.4 and loss tangent of 0.637 are used for water at $20 \mathrm{deg} \mathrm{C}$ and $12 \mathrm{GHz}$. These values for water ${ }^{1}$ agree closely with those published in $[6,7]$. The resulting calculated S-parameters of water films are shown in Table 3 for normal incidence at $12 \mathrm{GHz}$.

Next, the water film terminated by the fiberglass-screen reflector is considered. Through the use of S-parameters shown in Table 3, and a load with a reflection-coefficient magnitude of 0.9994 and phase angle of $123.9 \mathrm{deg}$, the input reflection coefficient at the surface of the water film is calculated from Eq. (1). The results show that the return losses are unexpectedly high. For example, for the waterlayer thickness of $0.10 \mathrm{~mm}(0.004 \mathrm{in}$.$) , the return loss is$ $12.8 \mathrm{~dB}$. This result is consistent with experimental observation of about $10-\mathrm{dB}$ signal losses when the fiberglass surfaces became wet from rain. ${ }^{2}$

To study the loss behavior in more detail, the return losses are shown plotted in Fig. 3 for water film terminated by a load with a reflection-coefficient magnitude of 0.9994 and a variable phase. Note that for the water-film thickness of $0.1 \mathrm{~mm}$, the return loss is about $13 \mathrm{~dB}$ when the load phase angle is about $124 \mathrm{deg}$, but the actual peak of the resonance curve is at a lower phase angle and is about $25 \mathrm{~dB}$. The exact values for the peaks of the curves for the various water-film thicknesses were calculated from Eq. (18) and are shown in Table 4. The high return losses result from the position of the maximum E-field having shifted to the inside of the film of water, which is very dissipative.

The values for minimum return losses are also tabulated in Table 4 , as well as the fiberglass-layer thicknesses that correspond to maximum and minimum return losses. It is of interest to note that to obtain minimum return loss, the thickness of the fiberglass layer should be $5.84 \mathrm{~mm}$ ( $0.230 \mathrm{in}$.). The corresponding load phase angle (represented by the fiberglass-screen reflector) would then be close to $180 \mathrm{deg}$. Except for small losses due to the dielectric, this condition would be equivalent to the reflector

\footnotetext{
${ }^{1}$ S. J. Keihm, private communication, Microwave Observational Systems Section, Jet Propulsion Laboratory, Pasadena, California, September 1991.

${ }^{2}$ D. A. Bathker, private communication, Ground Antennas and Facilities Engineering Section, Jet Propulsion Laboratory, Pasadena, California, July 1989.
} 
having no dielectric layer, where the rain film would lie directly on the metal surface. This result of a minimum return loss is consistent with experimental observations that very little degradation or signal loss occurs when rainwater wets a solid metallic reflector surface.

If the reflector were manufactured with a fiberglasslayer thickness of $5.84 \mathrm{~mm}(0.230 \mathrm{in}$.), rather than the current $1.59 \mathrm{~mm}(0.0625 \mathrm{in}$.), the return loss in dry conditions for the new thickness would be degraded only to $0.026 \mathrm{~dB}$ as compared to $0.005 \mathrm{~dB}$ for the original 1.59$\mathrm{mm}$ thickness. However, if the fiberglass surface were to become wet with water thicknesses varying from 0.05 to $0.10 \mathrm{~mm}$, the return loss would still be the $0.026-\mathrm{dB}$ worst case as compared to $12.8 \mathrm{~dB}$ for a fiberglass-layer thickness of $1.59 \mathrm{~mm}$.

The equations used for this study of a fiberglass-layered reflector can also be applied for performance studies of graphite-epoxy-layered reflector surfaces being proposed for future ground-space communication antennas. It is important that the thicknesses of the dielectric layer be designed properly so as to obtain good performance in wet conditions due to rain or condensation.

\section{Conclusions}

The analytical equations derived in this article are useful for determining worst-case return losses that can occur when a passive two-port network is terminated with a mismatched load. In one of the examples presented, the equations were useful for predicting worst-case return losses that could occur when water film was on a reflector surface that had a dielectric layer. The same type of analysis might be applied to a similar type of reflector painted with thermal diffusive paint suspected of being very lossy.

Although it was not shown through a specific example, for $\left(\left|\Gamma_{T}\right|<1\right)$, the equations presented in this article can also be used to design dielectric- or air-line spacers that can be inserted between a fixed load $\left(\left|\Gamma_{T}\right|<1\right)$ and the network if the goal is to obtain maximum or minimum return loss from the terminated two-port network.

\section{Acknowledgments}

The Ku-band reflector antenna resonance phenomenon in rainy conditions was brought to the author's attention by D. Bathker of the Ground Antennas and Facilities Engineering Section. Discussions with him motivated the studies and derivation of the equations presented in this article. A "Dielectric Sheet" computer program written in 1972 by Dr. C. Yeh, a consultant to JPL, was helpful in confirming the general case S-parameter equations presented in this article. His derivations were based on free-space considerations, rather than the network theory approach used in this article.

\section{References}

[1] D. M. Kerns and R. W. Beatty, Basic Theory of Waveguide Junctions and Introductory Microwave Network Analysis, New York: Pergamon, 1967.

[2] R. L. Fante and M. T. McCormack, "Reflection Properties of the Salisbury Screen," IEEE Trans. on Antennas and Propagation, vol. 36, no. 10, pp. 14431454, October 1988.

[3] T. Y. Otoshi, "Precision Reflectivity Loss Measurements of Perforated-Plate Mesh Materials by a Waveguide Technique," IEEE Trans. on Instrumentation and Measurement, vol. IM-21, pp. 451-457, November 1972. 
[4] K. Woo and T. Y. Otoshi, "Further RF Study of Reflector Surface Materials for Spacecraft Antennas," Space Programs Summary No. 36-65, vol. III, Jet Propulsion Laboratory, Pasadena, California, pp. 47-52, October 31, 1970.

[5] W. A. Imbriale, V. Galindo-Israel, and Y. Rahmat-Samii, "On the Reflectivity of Complex Mesh Surfaces," IEEE Trans. on Antennas and Propagation, vol. 39, no. 9, pp. 1352-1365, September 1991.

[6] D. S. Engelder and C. R. Buffler, "Measuring Dielectric Properties of Food Products at Microwave Frequencies," Microwave World, vol. 12, no. 2, Summer 1991.

[7] H. J. Liebe, G. A. Hufford, and T. Manabe, "A Model for the Complex Permittivity of Water at Frequencies Below $1 \mathrm{THz}$," International Journal of Infrared and Millimeter Waves, vol. 12, pp. 664, Table 1, November 7, 1991. (Note: $1 \mathrm{THz}$ $=1000 \mathrm{GHz}$.) 
Table 1. Measured S-parameters of the 64-m diameter antenna perforated panel test sample at $8.448 \mathrm{GHz}$, with perpendlcular polarlzation, 38.5-deg Incidence angle, and $\phi=0$ deg.

\begin{tabular}{ccc}
\hline S-parameter & Magnitude & Phase, deg \\
\hline$S_{11}$ & 0.9990 & 172.4 \\
$S_{21}$ & 0.0305 & 82.2 \\
\hline
\end{tabular}

Notes: $1 . S_{12}=S_{21}$ and $S_{22}=S_{11}$.

2. S-parameters are defined for free-space characteristic impedance $Z_{01}$ at the input and output ports.

3. Perforated-plate hole diameter, hole-to-hole spacing, with thicknesses of $4.763 \mathrm{~mm}(0.1875 \mathrm{in}),. 6.350 \mathrm{~mm}(0.250 \mathrm{in}$.), and $2.286 \mathrm{~mm}$ (0.090 in.).

Table 2. Load rellectlon coefilclent phase angle for minimum and maximum return losses for the 64-m dlameter antenna perforated plate (se0 Table 1).

\begin{tabular}{|c|c|c|c|}
\hline $\begin{array}{l}\Gamma_{L} \\
\text { magnitude }\end{array}$ & $\begin{array}{c}\Gamma_{L} \\
\text { phase, deg }\end{array}$ & $\begin{array}{l}\text { Return } \\
\text { loss, dB }\end{array}$ & Comments \\
\hline 1.0 & -172.40 & 33.21 & $\begin{array}{l}\text { This maximum return loss occurs when } \\
\text { the distance from a short }=0.4894 \lambda_{g}{ }^{a}\end{array}$ \\
\hline 1.0 & -4.00 & 0.0040 & $\begin{array}{l}\text { This minimum return loss occurs when } \\
\text { the distance from a short }=0.2556 \lambda_{g}\end{array}$ \\
\hline 0.5 & -172.27 & 0.1641 & Maximum return loss \\
\hline 0.5 & 8.80 & 0.0056 & Minimum return loss \\
\hline 0.1 & -172.07 & 0.0092 & Maximum return loss \\
\hline 0.1 & 8.09 & 0.0076 & Minimum return loss \\
\hline 0.0 & All values & 0.0087 & $20 \log 10\left|S_{11}\right|$ \\
\hline \multicolumn{4}{|c|}{$\frac{\lambda_{0}}{-\sin ^{2} \theta_{i}}=1.785^{\prime \prime}$} \\
\hline
\end{tabular}


Table 3. Calculated S-parameters of water film for relative permittlvity $=55.4$, loss tangent $=0.637$, at $12 \mathrm{GHz}$, and with normal Incldence angle.

\begin{tabular}{ccccccc}
\hline & \multicolumn{2}{c}{$0.05-\mathrm{mm}$ thick } & \multicolumn{2}{c}{$0.076-\mathrm{mm}$ thick } & \multicolumn{2}{c}{$0.10-\mathrm{mm}$ thick } \\
\cline { 2 - 6 } S-parameter & Magnitude & Phase, deg & Magnitude & Phase, deg & Magnitude & Phase, deg \\
\hline$S_{11}$ & 0.3249 & -139.48 & 0.4319 & -145.23 & 0.5137 & -149.82 \\
$S_{12}$ & 0.7855 & -16.56 & 0.6975 & -22.38 & 0.6239 & -27.07 \\
\hline
\end{tabular}

Notes: $1 . S_{12}=S_{21}$ and $S_{22}=S_{11}$.

2. S-parameters are defined for free-space characteristic impedance $Z_{01}$ at the input and output ports (see Fig. 1).

Table 4. Fiberglass plus screen load reflection coetflcients and corresponding return losses for varlous water-layer thicknesses whose S-parameters are shown In Table 3.

\begin{tabular}{ccccccr}
\hline $\begin{array}{c}\text { Fiberglass } \\
\text { thickness, } \\
\mathrm{mm}\end{array}$ & $\begin{array}{c}\text { Fiberglass } \\
\text { thickness, } \\
\text { in. }\end{array}$ & $\begin{array}{c}\Gamma_{L} \\
\text { magnitude }\end{array}$ & $\begin{array}{c}\Gamma_{L} \\
\text { phase, } \\
\mathrm{deg}\end{array}$ & $\begin{array}{c}\text { Return loss } \\
\text { (no water } \\
\text { layer), dB }\end{array}$ & $\begin{array}{c}\text { Water layer } \\
\text { thickness, } \\
\text { mm }\end{array}$ & $\begin{array}{c}\text { Return loss } \\
\text { (with water } \\
\text { layer), dB }\end{array}$ \\
\hline 1.59 & 0.0625 & 0.99940 & 123.86 & 0.0052 & 0.050 & 3.24 \\
1.59 & 0.0625 & 0.99940 & 123.86 & 0.0052 & 0.076 & 6.69 \\
1.59 & 0.0625 & 0.99940 & 123.86 & 0.0052 & 0.100 & 12.77 \\
\hline 2.344 & 0.0923 & 0.99700 & 70.22 & 0.0261 & 0.050 & 8.46 \\
2.075 & 0.0817 & 0.99824 & 93.37 & 0.0153 & 0.076 & 14.20 \\
1.836 & 0.0723 & 0.99895 & 109.83 & 0.0091 & 0.100 & 24.92 \\
\hline 5.865 & 0.2309 & 0.99704 & -179.32 & 0.0257 & 0.050 & 0.026 \\
5.847 & 0.2302 & 0.99704 & -178.81 & 0.0257 & 0.076 & 0.026 \\
5.834 & 0.2297 & 0.99704 & -178.44 & 0.0257 & 0.100 & 0.026
\end{tabular}

Note: $\Gamma_{L}$ is defined for free-space characteristic impedance $Z_{01}$ (see Fig. 1). 


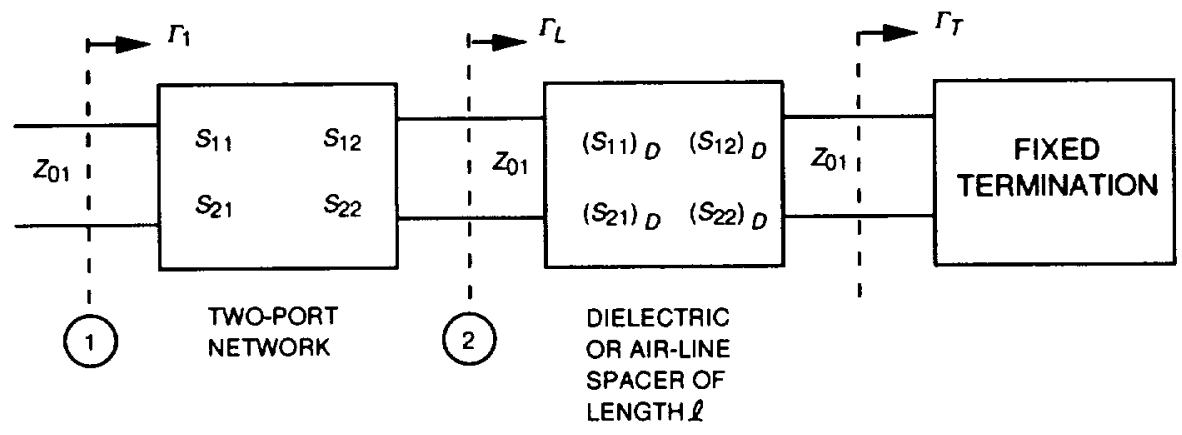

Fig. 1. Two-port network terminated In a $\Gamma_{L}$ load.

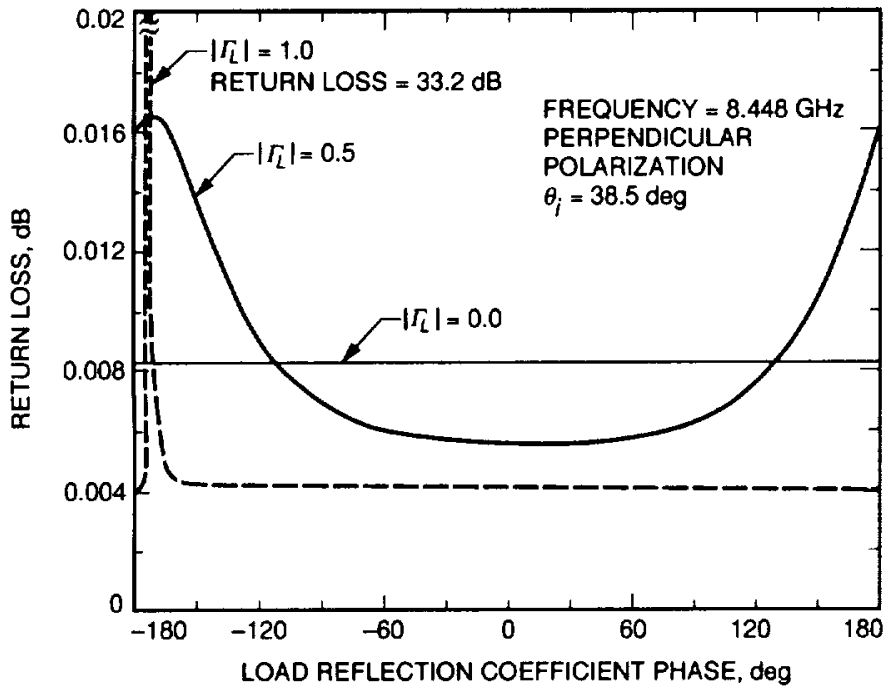

Fig. 2. Return loss of a perforated plate as a function of loadreflection coefficlent phase angle.

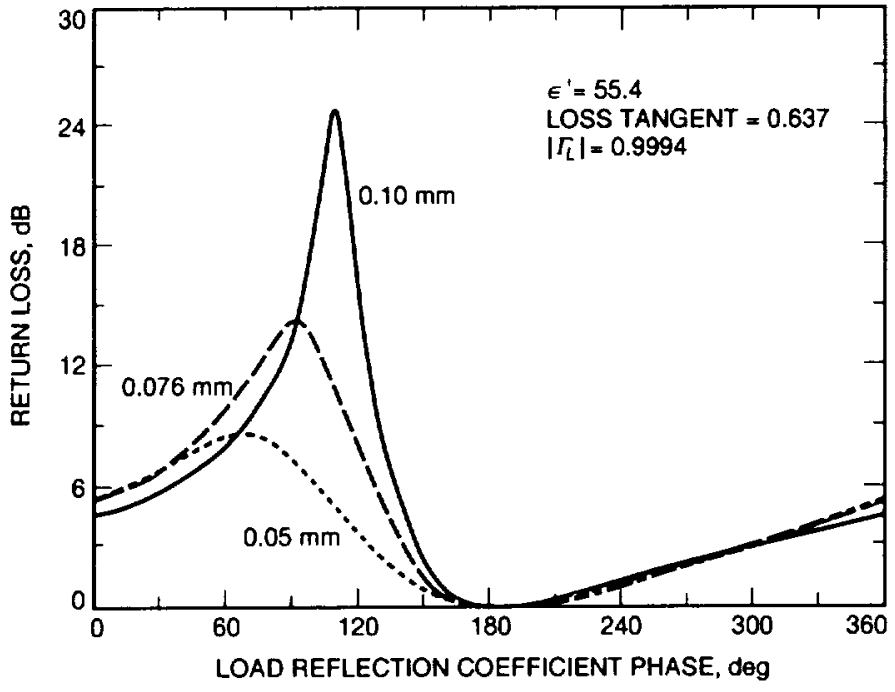

Fig. 3. Return loss versus load phase angle for various thicknesses of water films torminated in a load having a reflection coefficlent magnitude of 0.9994 at $12 \mathrm{GHz}$. 